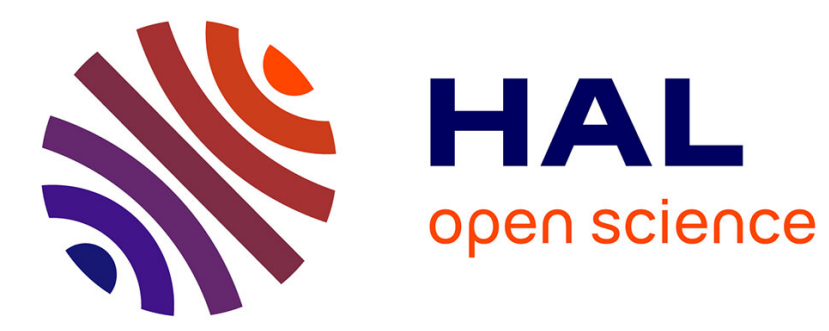

\title{
Probabilistic design for reliability in electronics and photonics : Role, significance, attributes, challenges
}

Ephraim Suhir, Alain Bensoussan, Golta Khatibi, Johann Nicolics

\section{To cite this version:}

Ephraim Suhir, Alain Bensoussan, Golta Khatibi, Johann Nicolics. Probabilistic design for reliability in electronics and photonics: Role, significance, attributes, challenges. IRPS 2015 (IEEE International Reliability Physics Symposium), Apr 2015, Monterey (CA), United States. hal-01698303

\section{HAL Id: hal-01698303 https://hal.science/hal-01698303}

Submitted on 1 Feb 2018

HAL is a multi-disciplinary open access archive for the deposit and dissemination of scientific research documents, whether they are published or not. The documents may come from teaching and research institutions in France or abroad, or from public or private research centers.
L'archive ouverte pluridisciplinaire $\mathbf{H A L}$, est destinée au dépôt et à la diffusion de documents scientifiques de niveau recherche, publiés ou non, émanant des établissements d'enseignement et de recherche français ou étrangers, des laboratoires publics ou privés. 


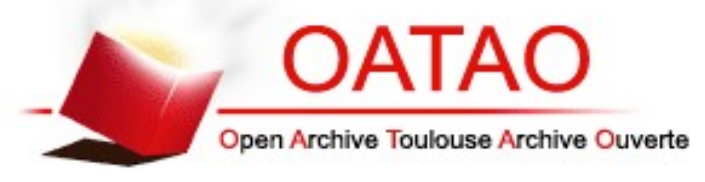

\section{Open Archive TOULOUSE Archive Ouverte (OATAO)}

OATAO is an open access repository that collects the work of Toulouse researchers and makes it freely available over the web where possible.

This is an author-deposited version published in : http://oatao.univ-toulouse.fr/ Eprints ID : 18036

\section{To link to this article :}

URL:http://ieeexplore.ieee.org/xpls/abs_all.jsp? arnumber $=7112749 \& \operatorname{tag}=1$ WOS : 000371888900082

To cite this version : Suhir, Ephraim and Bensoussan, Alain and Khatibi, Golta and Nicolics, Johann Probabilistic design for reliability in electronics and photonics : Role, significance, attributes, challenges. (2015) IRPS 2015 (IEEE International Reliability Physics Symposium).

Any correspondance concerning this service should be sent to the repository administrator: staff-oatao@listes-diff.inp-toulouse.fr 


\title{
Probabilistic Design for Reliability in Electronics and Photonics: Role, Significance, Attributes, Challenges
}

\author{
E. Suhir ${ }^{1,3}$, A. Bensoussan ${ }^{2}$, G. Khatibi ${ }^{3}$, J. Nicolics ${ }^{3}$ \\ ${ }^{1}$ Portland State University, Portland, OR, and \\ ERS Co., 727 Alvina Ct., Los Altos CA 94024, USA, 650-969-1530, suhire@aol.com, \\ 2 Institute of Technological Research, Toulouse, France, alain.bensoussan@irt-saintexupery.com, \\ ${ }^{3}$ Technical University, Vienna, Austria, golta.khatibi@tuwien.ac.at, johann.nicolics@,tuwien.ac.at
}

\section{ABSTRACT}

The recently suggested probabilistic design for reliability (PDfR) concept of electronics and photonics (EP) products is based on 1) highly focused and highly costeffective failure oriented accelerated testing (FOAT), aimed at understanding the physics of the anticipated failures and at quantifying, on the probabilistic basis, the outcome of FOAT conducted for the most vulnerable element(s) of the product of interest, for the most likely applications and for the most likely and meaningful combination of possible stressors (stimuli); 2) simple and physically meaningful predictive modeling (PM), both analytical and computeraided, aimed at bridging the gap between the obtained FOAT data and the most likely actual operation conditions; and 3) subsequent FOAT-and-PM-based sensitivity analysis (SA) using the methodologies and algorithms developed as important by-products at the two previous steps.

The PDfR concept proceeds from the recognition that nothing is perfect, and that the difference between a highly reliable and an insufficiently reliable product is "merely" in the level of the probability of its field failure. If this probability (evaluated for the anticipated loading conditions and the given time in operation) is not acceptable, then a SA can be effectively employed to determine what could/should be changed to improve the situation.

The PDfR analysis enables one also to check if the product is not "over-engineered", i.e., is not superfluously robust. If it is, it might be too costly. The operational reliability cannot be low, but it does not have to be higher than necessary either. It has to be adequate for the given product and application. When reliability and costeffectiveness are imperative, ability to optimize reliability is a must, and no optimization is possible if reliability is not quantified. We show that optimization of the total cost associated with creating a product with an adequate (high enough) reliability and acceptable (low enough) cost can be interpreted in terms of an adequate level of the availability criterion.

The major PDfR concepts are illustrated by practical examples. Although some advanced PDfR predictive modeling techniques have been recently developed, mostly for aerospace applications, the practical examples addressed in this talk employ more or less elementary analytical models. In this connection we elaborate on the roles and interaction of analytical (mathematical) and computer-aided (simulation) modeling.

We show also how the recently suggested powerful and flexible Boltzmann-Arrhenius-Zhurkov (BAZ) model and particularly its multi-parametric extension could be successfully employed to predict, quantify and assure operational reliability. The model can be effectively used to analyze and design EP products with the predicted, quantified, assured, and, if appropriate and cost-effective, even maintained and specified probability of operational failure.

It is concluded that these concepts and methodologies can be accepted as an effective means for the evaluation of the operational reliability of EP materials and products, and that the next generation of qualification testing (QT) specifications and practices for such products could be viewed and conducted as a quasi-FOAT, an early stage of FOAT that adequately replicates the initial non-destructive segment of the previously conducted comprehensive "fullscale" FOAT.

\section{INTRODUCTION}

Short- and especially long-term reliability of EP materials, devices and systems is the major challenge for broad application of EP products, especially in industries, such as aerospace, military, or long-haul communications, when high reliability is a must [1]. Here are some major problems and challenges envisioned and the most often and natural questions asked in connection with the reliability of the EP products:

- Qualification testing (QT) is the major means for making a viable EP device into a reliable product. It is well known, however, that the today's EP devices and systems that passed the existing QT often exhibit premature operational failures. Are the existing EP QT methodologies and procedures adequate [2]?

- Since many EP and especially optoelectronic (OE) products have been employed in industries that require failure-free operation (high reliability) for just several years, there are no well established and commonly accepted QT specifications, accelerated test (AT) methodologies and best practices for these products yet, and it is unclear whether the manufacturers should be shooting (perhaps unrealistically) for a 30 year long lifetime for an EP product or might be 
willing to settle for a shorter, but more or less satisfactorily substantiated and reasonably well predicted lifetime?

- $\quad$ The predicted (anticipated) reliability of an EP product should be different for different loading (stressors, stimuli) and for different fabrication technologies and applications [3]. How to consider and to quantify the effect of these conditions and applications, when planning the useful and cost-effective EP product lifetime [4]?

- EP materials degradation (aging) and failure mechanisms that have been found in actual operation were not always addressed and detected by the existing QT and other AT efforts $[5,6]$. How to establish the minimum list of the crucial ATs and the meaningful stressors, and, since the principle of superposition does not work in reliability engineering, how to establish the most important and physically meaningful combinations of these stressors?

- There is always a long way to go from what has been obtained in a lab at the research stage to a viable and reliable industrial product at the development and fabrication stages. How could this way be shortened for the EP products and for applications, where high reliability level is required?

- Since real time degradation (aging) is a slow process that might take years to manifest, could physically meaningful and cost-effective AT methodologies for measuring the degradation (aging) rates and consequences be considered, developed and implemented [7]?

- Predictive modeling (PM) has proven to be a highly useful and time- and cost-effective means for understanding the physics of failure and designing the most practical ATs in many areas of EP engineering [8-16]. There is certainly a need for developing such models, with an emphasis on validation, if possible, of the observed field failures. Which models might be the most needed ones: thermal, environmental, mechanical, radiation related, most likely combinations of these, anything else?

- The above concerns are primarily industries concerns, but what should be the major reliability related worries of a particular EP product manufacturer, especially when high and predictable reliability is imperative?

In the analysis that follow we do not try to answer all the above questions, but rather intend to partially address and discuss some of the formulated problems, with an objective to show how the recently suggested PDfR concept based on the highly focused and highly cost-effective FOAT and simple and physically meaningful PM techniques can be effectively used for making a viable EP device into a reliable product, with the predicted, quantified, assured, and, if appropriate and cost-effective, even maintained operational probability of failure $(\mathrm{PoF})$.

\section{WHEN RELIABILITY IS IMPERATIVE ABILITY TO QUANTIFY IT IS A MUST}

The ultimate broad goal of the EP industries is to make their deliverables consistently robust in the field by establishing industry wide QT methodologies and practices. QT is and should be the major means that the industries use to make their viable devices into reliable and sellable products. QT brings to a "common denominator" different EP manufacturers and different EP products. On the other hand, the short-term and practical goal of a particular EP product manufacturer is to conduct and pass the existing QT specifications and requirements without questioning if or to what extent they are adequate. Many EP products that passed the today's QTs have been known, however, to often prematurely fail in the field. Are the today's EP QT and specifications adequate? Do the EP manufacturing industries need new approaches to qualify their products? And if they do, could the existing EP specifications, procedures and practices be improved to an extent that if an EP product passed the existing QT, there is a quantifiable and sustainable way to assure that the product will satisfactorily perform in the field?

At the same time, there exists a perception, perhaps a rather substantiated one that some EP products or at least some important parts of these products are highly reliable and never fail. The very existence of such a perception could be attributed to the superfluous and unnecessary robustness of a particular product or a particular component for the given application. When failure occurs, root cause analyses are conducted; origins and causes of failures detected, and appropriate corrective actions are taken. Such actions are usually aimed at strengthening the design's weakest link, while the elements that did not fail remain unchanged. As a result, the product becomes overengineered and, very likely, too complex and too costly. Could it be proven that a particular EP product or an element that "never failed" is indeed superfluously robust and is therefore too costly for the application it is intended for? Is it possible that the manufacturer spent more time and expense than it was necessary to produce this product, and the customer paid too much to purchase it?

To find answers to these questions one has to find a consistent and a trustworthy way to quantify the product's reliability [17-19]. Then it would become possible not only to assure its adequate performance, but also to determine if a well understood and a well substantiated reduction in the superfluous reliability of the product could be effectively translated into considerable cost savings.

There is always an incentive to optimize reliability. Based on such an optimization, it would be possible to establish the best compromise between reliability, cost effectiveness and time-to-market (to completion) of the product of interest. EP products intended for high reliability applications are, as a rule, expensive, and therefore the ability to understand the relationship between the reliability and cost, and, if possible, to bring the total designfabrication-operational cost down without compromising reliability is of obvious importance. No optimization is possible if reliability is not quantified and evaluated with consideration of the expenses associated with the cost of 
creating a reliable product and the cost of its restoration, if failure occurs [7].

Last but perhaps the most important consideration in favor of an attempt to quantify operational reliability is that consistent and sustainable prediction and quantification of the operational reliability of an EP product would enable one to develop the most effective QT methodologies, procedures and specifications. These should consider the expected (required) time in operation, the most likely operation conditions, and possible consequences of failure.

Reliability is not an exact science, and sufficient or insufficient reliability is often, as they say, in the eye of the beholder. Intuition and vision often play a significant role in the reliability assessments and expectations. Quantification, however, enables one to minimize the level of subjectivity in reliability assessments and judgments and narrow the gap between the art and science of reliability engineering.

Let us show, e.g., using rather elementary reasoning, how the total cost of a product associated with reliability (dependability) on one hand and cost-effectiveness on the other could be minimized [7]. Let us assume that the cost of achieving and improving reliability of an EP product can be estimates based on an exponential formula

$$
C_{R}=C_{R}(0) \exp \left[r\left(R-R_{0}\right)\right],
$$

where $R=M T T F$ is the actual level of the MTTF, $R_{0}$ is the specified MTTF level, $C_{R}(0)$ is the cost of achieving the $R_{0}$ level of reliability and $r$ is the cost factor associated with reliability improvements. Similarly, let us assume that the cost of reliability restoration (repair) of the product of interest can be also assessed by an exponential formula

$$
C_{F}=C_{F}(0) \exp \left[-f\left(R-R_{0}\right)\right],
$$

where $C_{F}(0)$ is the cost of restoring the product's reliability, and $f$ is the factor of the reliability restoration (repair) cost. The latter formula reflects a natural assumption that the cost of repair is lower for a product of higher reliability. The total cost $C=C_{R}+C_{F}$ has its minimum

$$
C_{\min }=C_{R}\left(1+\frac{r}{f}\right)=C_{F}\left(1+\frac{f}{r}\right),
$$

when the minimization condition $r C_{R}=f C_{F}$ is fulfilled. Let us further assume that the factor $r$ of the reliability improvement cost is inversely proportional to the MTTF (dependability criterion), and the factor $f$ of the reliability restoration cost is inversely proportional to the mean time to repair MTTR (reparability criterion). With such a physically meaningful assumption we have:

$$
C_{\text {min }}=\frac{C_{R}}{K}=\frac{C_{F}}{1-K},
$$

where

$$
K=\frac{1}{1+\frac{\prec t_{r} \succ}{\prec t_{f} \succ}}=\frac{1}{1+\frac{M T T R}{M T T F}}
$$

is the availability, i.e., the probability that the product is sound and is available to the user any time at the steadystate operations. In the last formula $\prec t_{f} \succ=M T T F$, and $\prec t_{r} \succ=M T T R$ is the mean time to repair. The result obtained for the total minimum cost establishes, in an elementary way, the relationship between the minimum total cost of achieving and maintaining (restoring) the adequate reliability level and the availability criterion. The obtained relationship quantifies the intuitively obvious fact that the total cost of the product depends on both the total cost and the availability of the product. The formula $\frac{C_{F}}{C_{R}}=\frac{1}{K}-1$ that follows from the above derivation indicates that if the availability index $K$ is high, the ratio of the cost of repairs to the cost aimed at improved reliability is low. When the availability index is low, this ratio is high. Again, this intuitively obvious result is quantified by the obtained simple relationship. The above reasoning can be used, particularly, to interpret the availability index from the costeffectiveness point of view: the index $K=\frac{C_{R}}{C_{\text {min }}}$ reflects, in effect, the ratio of the cost of improving reliability to the minimum total cost of the product associated with its reliability level. This and similar, even elementary, models can be of help, particularly, when there is a need to minimize costs without compromising reliability, i.e., in various optimization analyses.

\section{DESIGN FOR RELIABILITY (DfR)}

Design for reliability (DfR) is, as is known, a set of approaches, methods and best practices that are supposed to be used at the design stage of the EP product to minimize the risk that the product might not meet the reliability objectives and customer expectations. "While $50 \%$ of the total actual cost of an electronic product is due to the cost of materials, $15 \%$ - to the cost of labor, $30 \%$ to the overhead costs and only $5 \%$ to the design effort, this effort influences about $70 \%$ of the total cost of the product. If reliability is taken care of at the design phase, the final cost of the product does not go up. If a reliability problem is detected during engineering the cost of the product goes up by a factor of 10. If the problem is caught in production phase, the cost of the product increases by a factor of 100 or more" [20].

Reliability is conceived at the design stage, whether one admits that or not. If the designer does a good job, there is a good chance that a "genetically healthy" EP product will be created. If this happens, then there are also high chances that subsequent reliability oriented or reliability affecting efforts conducted at different stages of the product's 
lifetime, such as fabrication, testing, maintenance, diagnostics, prognostics and health monitoring (PHM), will be successful as well. When deterministic (nonprobabilistic) DfR approach is used, reliability of a product could be based on the belief that sufficient reliability level will be assured if a high enough safety factor (SF) is used. The deterministic SF is defined as the ratio $S F=C / D$ of the capacity ("strength") $C$ of the product to the demand ("stress") $D$. In a particular problem the capacity and demand could be mechanical loads, elevated or low temperatures, electrical current, resistance, voltage, light intensity, humidity, etc. Cost effectiveness is seldom associated with the reliability level, if a deterministic approach is used.

\section{PROBABILISTIC DESIGN FOR RELIABILITY (PDfR)}

The SF (reliability assurance) determined as the ratio of the capacity (strength) to the demand (stress) cannot be used, when probabilistic design for reliability (PDfR) is considered, since both the capacity and the demand are random variables, and their ratio is a random variable as well. The PDfR SF is introduced as the ratio of the mean value $\prec \psi \succ$ of the safety margin $S M=\Psi=C-D$ to its standard deviation $\hat{\mathrm{s}}$, so that the probabilistic safety factor is evaluated as $S F=\frac{\prec \psi \succ}{\hat{S}}$. When the random time-tofailure (TTF) is of interest, the SF can be found as the ratio of the MTTF to the standard deviation of the TTF.

The use of SF as the measure of the probability of failure $(\mathrm{PoF})$ is more convenient than the direct use of the PoF itself. This is because this probability is expressed, for highly reliable and, hence, typical EP products, by a number, which is very close to one, and, for this reason, even significant changes in the product's design, which have an appreciable impact on its reliability, might have a minor effect on the level of the PoF, at least the way it appears to and perceived by the user. Indeed, if, e.g., the normal law is used to determine the probability of non-failure as the probability that the SM will remain higher than a certain level, then the SF will be 3.0901 for the probability of nonfailure of $0.999 ; 3.7194$ for the probability of non-failure of $0.9999 ; 4.5255$ for the probability of non-failure of 0.99999 ; and 4.7518 for the probability of non-failure of 0.999999 . The SF tends to infinity, when the probability of non-failure tends to one or the PoF tends to zero.

The PoF and/or the level of the $S F$ should be chosen depending on the existing experience, anticipated operation conditions, possible consequences of failure, acceptable risks, the available and trustworthy information about the capacity and the demand, the accuracy, with which the capacity and the demand are determined, possible costs and social benefits, information on the variability of materials and structural parameters, fabrication technologies and procedures, etc.

\section{TEN PDfR "COMMANDMENTS"}

PDfR concept [16-19] is central to calculate the PoF or the SF for an EP material or a product and to use this probability (and/or the probabilistic SF) as a suitable and physically most meaningful criterion of the product's performance. The following ten major principles ("commandments") reflect the rationale behind the PDfR concept:

1) PDfR concept is an effective means for improving the state-of-the-art in the field of the EP engineering by quantifying, on the probabilistic basis, the operational reliability of the product. The concept is based on the recognition that nobody and nothing is perfect, and that the difference between an unreliable product and a highly reliable one is "merely" in the level of their operational PoF; 2) The PoF of a product is never zero, but could and should be assessed (quantified) and brought to an acceptable (adequate) level;

3) The best EP product should be designed and fabricated as the best compromise between the needs (requirements) for its reliability, cost effectiveness and time-to-market (completion). These three major factors are of different importance, of course, for different applications and situations, but they are always of importance in any EP related technology. The most effective compromise can be established only if the PoF of an EP product is quantified;

4) EP product's reliability cannot be low, need not be higher than necessary, but has to be adequate for the given application, considering the expected and/or remaining useful lifetime (RUL), environmental conditions and possible consequences of failure;

5) Redundancy, trouble-shooting and maintenance are important factors to be considered, when adequate reliability level has to be maintained, especially if the "genetic health" of the product is not as high as necessary. As is known, redundancy enables one to create a highly reliable product out of relatively unreliable components;

6) When reliability is imperative, the ability to quantify it is highly desirable and is even a must, especially if one intends to optimize and to assure reliability, as far as its level and most feasible compromise with cost-effectiveness and timeto-market (completion) are concerned;

7) One cannot design a product with quantified, optimized and assured reliability by limiting the effort to the widely used today highly accelerated life testing (HALT) [21, 22]. HALT might detect and identify possible failure modes and even, to a limited (and unknown) extent, improve (ruggedize) reliability, but does not quantify it. One cannot quantify and assure reliability by simply following the existing qualification practices either, especially for new products and new applications, when such practices do not yet exist; 
8) Reliability is conceived at the design stage and should be taken care of, first of all, at this stage. It is at the design stage, when an attempt should be made to create a "genetically healthy" product. Reliability evaluations and assurances cannot be delayed until the product is fabricated, shipped to the customer and installed. It is too late at this stage to change the design or the materials for improved reliability. That is why, when high reliability is critical, users like NASA, whose EP products have to perform in a failure-free fashion in operation, have to re-qualify parts in order to assess their RUL, use redundancy to build a reliable enough system out of insufficiently reliable components and to employ and master various PHM technologies, instrumentations and techniques to maintain high level of operational performance of insufficiently "genetically" healthy materials, devices and systems. It is noteworthy that by underestimating the importance of the design stage and putting the emphasis on the PHM effort one might increase considerably the product's cost. It is also noteworthy that redundancy, PHM and other auxiliary health monitoring (managing) equipment and instrumentation is not $100 \%$ reliable either. The reliability engineer should therefore consider this circumstance when addressing the long-term reliability of the EP product;

9) Highly cost-effective and highly focused FOAT geared to a limited number of pre-determined simple, easy-to-use and physically meaningful predictive reliability models and aimed at understanding the physics of failure that is anticipated and quantified by these models is an important constituent part of the PDfR effort;

10) Predictive modeling (PM), not necessarily using the well known FOAT models, is another important constituent of the PDfR approach. PM, in combination with wellestablished FOAT models, is a powerful means to carry out, if necessary, sensitivity analyses (SA) with an objective to quantify and practically nearly eliminate failures by making the PoF sufficiently low; this principle is referred to some time as the "principle of practical confidence".

\section{ACCELERATED TESTING (AT)}

Shortening of product's design and development time does not allow in the today's industrial environment for time consuming reliability investigations. To get maximum reliability information in minimum time and at minimum cost is the major goal of a manufacturer. At the same time, it is impractical to wait for failures, when the lifetime of a typical today's EP product is hundreds of thousands of hours. AT is therefore both a must and a powerful means in EP manufacturing. The major AT types are summarized in Table 1 at the end of this paper.

Product development testing (PDT) is a crucial part of the DfR effort. A typical example is shear-off testing when there is a need to determine the most feasible bonding material and its thickness.

HALT (see, e.g., [21]) is currently widely employed, in different modifications, with an intent to determine the product's reliability weaknesses, assess its reliability limits, ruggedize the product by applying elevated stresses (not necessarily mechanical and not necessarily limited to the anticipated field stresses) that could cause field failures, and to provide large (although, actually, unknown) SMs (not SFs!) over expected in-use conditions. HALT often involves step-wise stressing, rapid thermal transitions, and other means that enable one to carry out testing in a time and cost effective fashion. HALT is sometimes referred to as a "discovery" test. It is not a QT though.

QT is the "pass/fail" test and, as such, is the major means for making a promising and viable EP device into a reliable and marketable product. QT is a must in EP fabrication and reliability assurance. It brings to a "common denominator" different manufacturers and different products.

When it comes to manufacturing, mass fabrication generates, in addition to desirable-and-robust ("strong") products, also some amount of undesirable-and-unreliable ("weak") devices ("freaks"), which, if shipped to the customer, will most likely fail in the field. Burn-in AT (BIT) is supposed to detect and to eliminate such "freaks". As a result, the final bathtub curve of a product that successfully underwent BIT is not supposed to contain the infant mortality portion. In the today's practice, BIT, which is a destructive test (FOAT) for the "freaks" and a nondestructive test for the healthy devices, is often run within the framework of and concurrently with HALT.

\section{FOAT AS AN EXTENSION OF HALT}

A highly focused and highly cost effective FOAT [22, 23 ] is the "heart" of the PDfR concept. FOAT should be conducted in addition to and, in some cases, even instead of HALT. FOAT is a solid experimental foundation of the PDfR approach. Predictions, based on the FOAT and subsequent PM, might not be perfect, at least at the beginning, but it is still better to pursue this effort rather than to turn a blind eye on the fact that there is always a non-zero probability of the product's failure.

Understanding the underlying reliability physics for the EP product performance is critical. If one sets out to understand the physics of failure in an attempt to create a failure-free product (in accordance with the "principle of practical confidence") conducting a FOAT type of an experiment is imperative. FOAT's objective is to confirm the usage of a particular more or less well established PM, to confirm (say, after HALT is conducted) the physics of failure, and establish the numerical characteristics (activation energy, time constant, sensitivity factors, etc.) of the particular FOAT reliability PM of interest.

FOAT could be viewed as an extension of HALT. HALT is a "black box", i.e., a methodology which can be perceived in terms of its inputs and outputs without a clear knowledge of the underlying physics and the likelihood of failure. FOAT, on the other hand, is a "white box", whose main objective is to confirm usage of a particular PM that 
reflects a specific anticipated failure mode. The major assumption is, of course, that this model should be valid in both AT and in actual operation conditions.

While HALT does not measure (does not quantify) reliability. FOAT does. HALT can be used for "rough tuning" of an OE product's reliability. FOAT should be employed when "fine tuning" is needed, i.e., when there is a need to quantify, assure and even specify the operational reliability of an EP product. HALT tries, quite often rather successfully, to "kill many unknown birds with one (also not very well known) stone".

HALT has demonstrated, however, over the years its ability to improve robustness through a "test-fail-fix" process, in which the applied stresses (stimuli) are somewhat above the specified operating limits. This "somewhat above" is based, however, on the intuition, rather than on calculations. There is a general perception nevertheless that HALT might be able to quickly precipitate and identify failures of different origins.

FOAT and HALT could be carried out separately, or might be partially combined in a particular AT effort. Since the principle of superposition does not work in reliability engineering, both HALT and FOAT use, when appropriate, combined stressing under various stimuli (stressors).

New products present natural reliability concerns, as well as significant challenges at all the stages of their design, manufacture and use. An appropriate combination of HALT and FOAT efforts could be especially useful for ruggedizing and quantifying reliability of such products. It is always necessary to correctly identify the expected failure modes and mechanisms, and to establish the appropriate stress limits of HALTs and FOATs with an objective to prevent "shifts" in the dominant failure mechanisms. There are many ways of how this could be done (see, e.g. [24, 25]). The FOAT based approach could be viewed as a quantified and reliability physics oriented HALT.

FOAT should be implemented, whenever feasible and appropriate, in addition to HALT. In some cases FOAT could be implemented even instead of HALT, especially for new products, whose operational reliability is unclear and for which no experience is accumulated and no best practices exist. The FOAT approach should be geared to a particular technology and application, with consideration of the most likely stressors.

\section{SOME KNOWN FOAT MODELS}

Here are some known and more or less widely used FOAT models [17, 19, 22]:

- Arrhenius' equation

$$
\tau=\tau_{0} \exp \left(\frac{U}{k T}\right)
$$

Here $\tau$ is the lifetime, $\tau_{0}$ is the time constant, $U$ is the activation energy, $T$ is the absolute temperature and $k$ is Boltzmann's constant. This equation and its numerous extensions and modifications are used when there is evidence or belief that the elevated temperature is the major cause of the product's degradation and failure. Examples are lifetime of electrical insulations and dielectrics; solid state and semiconductor materials and devices; inter-metallic diffusion; lifetime of batteries and solar cells, lubricants; greases, thermal interface materials, plastics, etc. Arrhenius model can be used also when evaluating reliability characteristics other than lifetime, such as, e.g., leakage current or light output.

- BAZ model [26-30]

$$
\tau=\tau_{0} \exp \left(\frac{U_{0}-\gamma \sigma}{k T}\right)
$$

can be used when the material or the device experience combined action of elevated temperature $T$ and external loading $\sigma$ (not necessarily mechanical). Although in Zhurkov's tests the loading $\sigma$ was a constant mechanical tensile stress, it has been recently suggested [17-19] that any other stimulus of importance (voltage, current, thermal stress, humidity, radiation, etc.) can be used as such a stress. The effective activation energy

$$
U=k T \ln \frac{\tau}{\tau_{0}}=U_{0}-\gamma \sigma
$$

plays in the BAZ model the role of the stress-free energy $U_{0}$ in the Arrhenius model (1). The BAZ model and the Arrhenius equation can be obtained as the steady-state solution to the Fokker-Planck equation in the theory of Markovian processes [28]. It has been shown that this solution represents the worst case scenario for the Markovian process addressed and that the reliability predictions based on the BAZ model are reasonably conservative and are advisable therefore to be used in engineering practice.

- Various and numerous crack growth models are used to assess the fracture toughness of materials in the brittle state, and to assess the rate of crack propagation. It is noteworthy that the today's fracture mechanics is able to predict crack propagation, but not crack initialization. It is presumed that all the materials have cracks, but if these cracks are short and randomly oriented, they will most likely not propagate.

- Inverse power law is used in numerous modifications of the Coffin-Manson's semi-empirical relationships aimed at the prediction of the low cycle fatigue life-time of solders that operate above the yield limit.

- Miner-Palmgren rule is used to address fatigue when the elastic limit is not exceeded.

- Weakest link models are used to evaluate the lifetime in extremely brittle materials, like $\mathrm{Si}$ or compound semiconductors, with highly localized defects.

- Stress-strength interference models are widely used in various problems of structural (physical) design in many areas of engineering, including microelectronics. Typically it is assumed that the distributions of the demand (stress) 
and capacity (strength) do not change with time, but, if they do (which is typical for OE products), an appropriate modification of these models could be considered;

- $\quad$ Peck's model

$$
\tau=\tau_{0} H^{-n} \exp \left(\frac{U_{0}}{k T}\right)
$$

considers the combined action of the elevated temperature and relative humidity $H$.

- Peck's power law

$$
\tau=\tau_{0} H^{-n} f(V) \exp \left(\frac{U_{0}}{k T}\right)
$$

considers also the applied voltage.

- Eyring formula could be obtained from (4) by substituting the relative humidity $H$ in it with the peak current $I$.

It is important to point out that the model (2) has a solid physical meaning, reflected by the level of the stress-free and effective (stress dependent) activation energy, while the formulas (4) and (5) are semi-empirical. On the other hand, if testing is carried out based, say, on the formula (4), the results can be easily interpreted in terms of the model (2). Indeed, by putting $\sigma=H$ in the BAZ formula (2) and equating the lifetimes predicted by the formulas (2) and (4), we obtain:

$$
\gamma=n k T \frac{\ln H}{H}
$$

- Black's equation can be obtained by substituting in the Peck's model (4) the relative humidity $H$ with the current density $j$. The equation is used to quantify the reliability in electro-migration problems, evaluate the lifetime of heterojunction in bipolar transistors and, in some cases, to account for the role of humidity as well.

It is important to emphasize that all these models can be interpreted in terms of the probability of failure under the given loading conditions and after the given time in operation.

A bathtub curve is a good example of a FOAT model and could be viewed as a reliability "passport" of an EP product of interest. If this curve is available, then many useful quantitative predictions could be made. E.g., the reliability physics related degradation (aging) process can be evaluated as the difference between the ordinates of the experimentally obtained bathtub curve and theoretically predicted ordinates of the statistical failure rates for mass produced products [31]. The latter process decreases with time, while the degradation process increases with time. The statistical process prevails at the infant mortality portion of the curve, while the degradation process prevails at its wear out portion. At the steady-state portion these two processes compensate for each other and result in a more or less constant failure rate.

\section{SOME SIMPLE PDfR EXAMPLES}

\subsection{Adequate heat sink}

Consider a device whose steady-state operation is determined by the Arrhenius equation (1). The probability of non-failure can be found using the exponential law of reliability as

$$
P=\exp \left[-\frac{t}{\tau_{0}} \exp \left(-\frac{U}{k T}\right)\right]
$$

Solving this equation for the absolute temperature $T$, we have:

$$
T=-\frac{U / k}{\ln \left(-\frac{\tau_{0}}{t} \ln P\right)} .
$$

Addressing, e.g., surface charge accumulation failure, for which the ratio of the activation energy to the Boltzmann's constant is $\frac{U}{k}=11600^{\circ} \mathrm{K}$, assuming that the FOAT- predicted time factor $\tau_{0}$ is $\tau_{0}=2 \times 10^{-5}$ hours, that the customer requires that the probability of failure at the end of the device's service time of $t=40,000$ hours does not exceed $Q=10^{-5}$, the formula (8) results in the following allowable temperature: $T=352.3^{0} \mathrm{~K}=79.3^{0} \mathrm{C}$. Thus, the heat sink should be designed accordingly, and the vendor should be able to deliver such a heat sink. The situation changes to the worse, if the temperature of the device changes, especially in a random fashion, but this situation can also be predicted by a simple probabilistic analysis, which is, however, beyond the scope of this paper.

\subsection{Reliable seal glass [32]}

The maximum interfacial shearing stress in the thin solder glass layer can be computed by the formula: $\tau_{\max }=k h_{g} \sigma_{\max }$. Here $k=\sqrt{\frac{\lambda}{\kappa}}$ is the parameter of the interfacial shearing stress, $\lambda=\frac{1-v_{c}}{E_{c} h_{c}}+\frac{1-v_{g}}{E_{g} h_{g}}$ is the longitudinal axial compliance of the ceramics-glass assembly, $\quad \kappa=\frac{h_{c}}{3 G_{c}}+\frac{h_{g}}{3 G_{g}}$ is the longitudinal interfacial compliance of this assembly, $G_{c}=\frac{E_{c}}{2\left(1+v_{c}\right)}$ and $G_{g}=\frac{E_{g}}{2\left(1+v_{g}\right)}$ are the shear moduli of the ceramics and 
glass materials, $\sigma_{\max }=\frac{\Delta \alpha \Delta t}{\lambda h_{g}}$ is the maximum normal stress in the midportion of the glass layer, $\Delta t$ is the change in temperature from the soldering temperature to the room or testing temperature, $\Delta \alpha=\bar{\alpha}_{c}-\bar{\alpha}_{g}$ is the difference in the effective coefficients of thermal expansion/contraction (CTEs) of the ceramics and the glass materials, $\bar{\alpha}_{c, g}=\frac{1}{\Delta t} \int_{t}^{t_{0}} \alpha_{c, g}(t) d t$ are these coefficients for the given temperature $t, t_{0}$ is the annealing (zero stress, set up) temperature, and $\alpha_{c, g}(t)$ are the time dependent CTEs for the materials in question. In an approximate analysis one could assume that the axial compliance $\lambda$ of the ceramicsglass assembly is due to the glass only, so that the axial compliance of the assembly can be determined as $\lambda \approx \frac{1-v_{g}}{E_{g} h_{g}}$ and therefore the maximum normal stress in the solder glass can be evaluated by the well known formula $\sigma_{\max }=\frac{E_{g}}{1-v_{g}} \Delta \alpha \Delta t$ for a thin film (seal glass) fabricated on a thick substrate (ceramics).

While the geometric characteristics of the assembly, the change in temperature and the elastic constants of the materials can be determined with high accuracy, the certainty in the prediction of the difference in the CTEs of the brittle materials of the glass and the ceramics is not as good, to say the least. In addition, because of the obvious incentive to minimize the difference in the CTEs of the two materials, such a mismatch is characterized by a small difference of quite close numbers. This contributes to the additional uncertainty in the evaluation of this difference, and in additional justification of the application of the probabilistic approach.

Treating the CTEs of the two materials as normally distributed random variables, we intend to evaluate the probability $P$ that the thermal interfacial shearing stress is compressive (negative) and does not exceed a certain allowable level. Since this stress is proportional to the normal stress in the glass layer, and the normal stress is proportional to the difference $\Psi=\alpha_{c}-\alpha_{g}$ of the CTE of the ceramics and the glass materials, one wants to make sure that the requirement

$$
0 \leq \Psi \leq \Psi_{*}=\frac{\sigma_{a}}{E_{g}} \frac{1-v_{g}}{\Delta t}
$$

takes place with a very high probability. This requirement actually means that the normal stress in the glass is compressive (negative) and does not exceed a certain allowable level, because if it does, the corresponding interfacial shearing stress might be too high to be acceptable. For normally distributed random variables $\alpha_{c}$ and $\alpha_{g}$ we conclude that the random variable $\Psi$ is also distributed in accordance with the normal law with the mean value $\prec \psi \succ=\prec \alpha_{c} \succ-\prec \alpha_{g} \succ$ and standard deviation $\sqrt{D_{\psi}}=\sqrt{D_{c}+D_{g}}$. Here $\prec \alpha_{c} \succ$ and $\prec \alpha_{g} \succ$ are the mean values of the materials CTEs, and $D_{c}$ and $D_{g}$ are their variances. The probability that the above governing inequality takes place can be found as

$$
P=\int_{0}^{\psi_{*}} f_{\psi}(\psi) d \psi=\Phi_{1}\left(\gamma^{*}-\gamma\right)-\left[1-\Phi_{1}(\gamma)\right],
$$

where

$$
\Phi_{1}(t)=e r f t=\frac{1}{\sqrt{2 \pi}} \int_{-\infty}^{t} e^{-t^{2} / 2} d t
$$

is the error function, $\gamma=\frac{\prec \psi \succ}{\sqrt{D_{\psi}}}$ is the SF for the CTE difference and $\gamma^{*}=\frac{\psi^{*}}{\sqrt{D_{\psi}}}$ is the SF with respect to the acceptable level of the allowable stress. Let, e.g., the elastic constants of the solder glass are $E_{g}=0.66 \times 10^{6} \mathrm{~kg} / \mathrm{cm}^{2}$ and $v_{g}=0.27$, the sealing (fabrication) temperature is $485^{\circ} \mathrm{C}$, the lowest (testing) temperature is $-65^{\circ} \mathrm{C}$ (so that $\Delta t=550^{\circ} \mathrm{C}$ ), the computed effective CTE's at this temperature are $\quad \bar{\alpha}_{g}=6.75 \times 10^{-6} 1 /{ }^{0} \mathrm{C}$ and $\bar{\alpha}_{c}=7.20 \times 10^{-6} 1 /{ }^{0} C$, the standard deviations of these STEs are $\sqrt{D_{c}}=\sqrt{D_{g}}=0.25 \times 10^{-6} 1 /{ }^{0} \mathrm{C}$ and the (experimentally obtained) ultimate compressive strength for the glass material is $\sigma_{u}=5500 \mathrm{~kg} / \mathrm{cm}^{2}$. With the SF of, say, 4 , we have $\sigma^{*}=\sigma_{u} / 4=1375 \mathrm{~kg} / \mathrm{cm}^{2}$. The allowable level of the parameter $\psi$ is therefore $\psi_{*}=\frac{\sigma_{a}}{E_{g}} \frac{1-v_{g}}{\Delta t}=\frac{1375}{0.66 \times 10^{6}} \frac{0.73}{550}=2.765 \times 10^{-6} 1 /{ }^{0} \mathrm{C}$. The mean value of the parameter $\psi$ is $\prec \psi \succ=\prec \alpha_{c} \succ-\prec \alpha_{g} \succ=0.450 \times 10^{-6} 1 /{ }^{0} C$, and its variance is 


$$
D_{\psi}=D_{c}+D_{g}=0.25 \times 10^{-12}\left(1 /{ }^{0} C\right)^{2} .
$$

Then the computed SFs are $\gamma=1.2726$ and $\gamma^{*}=7.8201$, and the predicted probability of non-failure of the seal glass material is

$$
P=\Phi_{1}\left(\gamma^{*}-\gamma\right)-\left[1-\Phi_{1}(\gamma)\right]=0.898 \text {. }
$$

Note that if the standard deviations of the materials CTEs were just $\sqrt{D_{c}}=\sqrt{D_{g}}=0.1 \times 10^{-6} 1 /{ }^{0} \mathrm{C}$, then the SFs would be much higher: $\gamma=3.1825$ and $\gamma^{*}=19.5559$, and the probability of non-failure would be as high as $P=0.999$.

\subsection{Extreme response [30]}

Let an EP component be operated in thermal cycling conditions, and the random amplitude of the induced stress, when a single cycle is applied, is distributed in accordance with the Rayleigh law

$$
f(r)=\frac{r}{D_{x}} \exp \left(-\frac{r^{2}}{2 D_{x}}\right) .
$$

Let us determine the most likely extreme value of the stress amplitude for a large number of cycles. The probability distribution density function $g\left(y_{n}\right)$ and the probability distribution function $G\left(y_{n}\right)$ for the extreme response $Y_{n}$ of the stress amplitude can be found as

$$
\begin{array}{r}
g\left(y_{n}\right)=n\left\{f(x)[F(x)]^{n-1}\right\}_{x=y_{n}} \\
G\left(y_{n}\right)=\left\{[F(x)]^{n}\right\}_{x=e_{n}} .
\end{array}
$$

From (9) and the first formula in (10) we obtain:

$$
g\left(y_{n}\right)=n \varsigma_{n}^{2} \exp \left(-\frac{\zeta_{n}^{2}}{2}\right)\left[1-\exp \left(-\frac{\zeta_{n}^{2}}{2}\right)\right]^{n-1} .
$$

Here $\varsigma_{n}=\frac{y_{n}}{\sqrt{D_{x}}}$ is the dimensionless stress amplitude value. The condition $g^{\prime}\left(y_{n}\right)=0$ yields:

$$
\varsigma_{n}^{2}\left[n \exp \left(-\frac{\zeta_{n}^{2}}{2}\right)-1\right]-\left[\exp \left(-\frac{\zeta_{n}^{2}}{2}\right)-1\right]=0 \text {. }
$$

If the number $n$ is large, the second term in the obtained expression is much smaller than the first one. Then we have:

$$
n \exp \left(-\frac{\varsigma_{n}^{2}}{2}\right)-1=0
$$

and therefore

$$
y_{n}=\varsigma_{n} \sqrt{D_{x}}=\sqrt{2 D_{x} \ln n}
$$

As evident from this result, the ratio of the extreme response $y_{n}$, after $n$ cycles are applied, to the maximum response $\sqrt{D_{x}}$, when a single cycle is applied, is $\sqrt{2 \ln n}$. This ratio is 3.2552 for 200 cycles, 3.7169 for 1000 cycles, and 4.1273 for 5000 cycles.

More complicated examples of FOAT and design decisions based on FOAT data could be found in [30].

\section{MULTI-PARAMETRIC BAZ MODEL \\ 10.1. Multi-parametric BAZ model}

Let the lifetime $\tau$ in the BAZ model (2) is viewed as the MTTF. Such an assumption suggests that if the exponential law of probability $P=\exp (-\lambda t)$ of nonfailure is used, the MTTF corresponds to the moment of time when the entropy of this law reaches its maximum value. Indeed, from the formula $H(P)=-P \ln P$ we obtain that the maximum value of the entropy $H(P)$ is equal to $e^{-1}$ and takes place for $P=e^{-1}=0.3679$. With this probability of non-failure, the formula (7) yields:

$t=\tau_{0} \exp \left(\frac{U}{k T}\right)$. Comparing this result with the Arrhenius equation (1) we conclude that the MTTF expressed by this equation corresponds to the moment of time when the entropy of the time-depending process $P=P(t)$ is the largest.

Let us elaborate on the substance of the multi-parametric BAZ model using an example of a situation when the EP product of interest is subjected to the combined action of the elevated relative humidity $H$ and elevated voltage $V$. Let us assume that the failure rate of an EP product, which characterizes the degree of propensity of the material or the device to failure, is determined by the level of the leakage current: $\lambda=\gamma_{I} I$. Then, using the type (2) model, one can seek the probability of the product's non-failure as

$$
P=\exp \left[-\gamma_{I} I t \exp \left(-\frac{U_{0}-\gamma_{H} H-\gamma_{V} V}{k T}\right)\right] .
$$

Here the $\gamma$ factors reflect the sensitivities of the device to the change in the corresponding stressors. Although only two stressors are selected - the relative humidity $H$ and the elevated voltage $V$ - the model can be easily made multi- 
parametric, i.e., generalized for as many stimuli as necessary.

The sensitivity factors $\gamma$ should be determined from the FOAT when the combined action of all the stimuli (stressors) of importance is considered. Because of that the structure of the multi-parametric BAZ should not be interpreted as a superposition of the effects of different stressors (as is known, superposition principle does not work in reliability engineering), but rather as a convenient and physically meaningful representation of the FOAT data.

The physical meaning of the distribution (12) could be seen from the formulas

$$
\begin{gathered}
\frac{\partial P}{\partial I}=-\frac{H(P)}{I}, \frac{\partial P}{\partial t}=-\frac{H(P)}{t}, \frac{\partial P}{\partial U_{0}}=\frac{H(P)}{k T}, \\
\frac{\partial P}{\partial H}=-\frac{H(P)}{k T} \gamma_{H}=-\gamma_{H} \frac{\partial P}{\partial U_{0}}, \\
\frac{\partial P}{\partial V}=-\frac{H(P)}{k T} \gamma_{V}=-\gamma_{V} \frac{\partial P}{\partial U_{0}},
\end{gathered}
$$

where $H(P)=-P \ln P$ is the entropy of the probability of non-failure. The formulas (13) can be obtained from (12) by differentiation.

The following conclusions can be made based on these formulas:

- The change in the probability of non-failure always increases with an increase in the entropy (uncertainty) of the distribution.

- This probability decreases with an increase in the leakage current and with time, which certainly makes physical sense.

- The last two formulas in (13) show the physical meaning of the sensitivity factors $\gamma$ : they can be found as the ratios of the change in the probability of non-failure with respect to the corresponding stimuli to the change of this probability with the change in the stress-free activation energy.

The equation (12) contains four empirical parameters that characterize the EP product of interest: the stress-free activation energy $U_{0}$ and three sensitivity factors $\gamma$ : leakage current factor, relative humidity factor and elevated voltage factor. Here is how these factors could be obtained from the highly focused and highly cost effective FOAT data.

First one should run the FOAT for two different temperatures $T_{1}$ and $T_{2}$, keeping the levels, low or high, of the relative humidity $H$ and elevated voltage $V$ the same in both tests; recording the percentages (values) $P_{1}$ and $P_{2}$ of non-failed samples (or values $Q_{1}=1-P_{1}$ and $Q_{2}=1-P_{2}$ of the failed samples); assuming a certain criterion of failure (say, when the level of the measured leakage current exceeds a certain level $I_{*}$ ), we obtain the following two relationships:

$$
\begin{aligned}
& P_{1}=\exp \left[-\gamma_{I} I_{*} t_{1} \exp \left(-\frac{U_{0}-\gamma_{H} H-\gamma_{V} V}{k T_{1}}\right)\right], \\
& P_{2}=\exp \left[-\gamma_{I} I_{*} t_{2} \exp \left(-\frac{U_{0}-\gamma_{H} H-\gamma_{V} V}{k T_{2}}\right)\right] .
\end{aligned}
$$

Since the numerators $U_{0}-\gamma_{H} H-\gamma_{V} V$ in these relationships are kept the same, the following transcendental equation must be fulfilled for the sought sensitivity factor $\gamma_{I}$ of the leakage current:

$f\left(\gamma_{I}\right)=\ln \left(-\frac{\ln P_{1}}{I_{*} t_{1} \gamma_{I}}\right)-\frac{T_{2}}{T_{1}} \ln \left(-\frac{\ln P_{2}}{I_{*} t_{2} \gamma_{I}}\right)=0$.

Here $t_{1}$ and $t_{2}$ are the times, at which the failures were detected. It is expected that more than just two series of FOAT tests and at more than two temperature levels are conducted, so that the sensitivity parameter $\gamma_{I}$ could be predicted with a high enough degree of accuracy (certainty).

At the second step, FOAT tests at two relative humidity levels $H_{1}$ and $H_{2}$ should be conducted for the same temperature and voltage. This leads to the relationship: $\gamma_{H}=\frac{k T}{H_{1}-H_{2}}\left[\ln \left(-\frac{\ln P_{1}}{I_{*} t_{1} \gamma_{I}}\right)-\ln \left(-\frac{\ln P_{2}}{I_{*} t_{2} \gamma_{I}}\right)\right]$

Similarly, by changing the voltages $V_{1}$ and $V_{2}$ at the next step of FOAT tests one could find:

$\gamma_{V}=\frac{k T}{V_{1}-V_{2}}\left[\ln \left(-\frac{\ln P_{1}}{I_{*} t_{1} \gamma_{I}}\right)-\ln \left(-\frac{\ln P_{2}}{I_{*} t_{2} \gamma_{I}}\right)\right]$

Finally, the stress-free activation energy can be computed as

$U_{0}=\gamma_{H} H+\gamma_{V} V-k T \ln \left(-\frac{\ln P}{I_{*} t \gamma_{I}}\right)$

for any consistent humidity, voltage, temperature and time.

The above relationships could be obtained particularly also for the case of zero voltage, i.e., without a high-voltage bias. This will provide additional information of the materials and device reliability characteristics.

\subsection{Numerical example}

Let, e.g., the following input information is available:

1) After $t_{1}=35 \mathrm{~h}$ of testing at the temperature $T_{1}=60^{\circ} \mathrm{C}=333^{\circ} \mathrm{K}$, the voltage $\mathrm{V}=600 \mathrm{~V}$ and the relative humidity $\mathrm{H}=0.85,10 \%$ of the tested modules exceeded the allowable (critical) level of the 
leakage current of $I_{*}=3.5 \mu \mathrm{A}$ and, hence, failed, so that the probability of non-failure is $P_{1}=0.9$;

2) After $t_{2}=70 \mathrm{~h}$ of testing at the temperature $T_{2}=85^{\circ} \mathrm{C}=358^{\circ} \mathrm{K}$ at the same voltage and the same relative humidity, $20 \%$ of the tested samples reached or exceeded the critical level of the leakage current and, hence, failed, so that the probability of non-failure is $P_{2}=0.8$.

Then the equation (12) results in the following transcendental equation for the leakage current sensitivity factor $\gamma_{I}$ :

$$
\begin{aligned}
& f\left(\gamma_{I}\right)=\ln \left(\frac{0.10536}{\gamma_{I}}\right)- \\
& -1.075075 \times \ln \left(-\frac{.0 .22314}{\gamma_{I}}\right)=0
\end{aligned}
$$

This equation has the solution $\gamma_{I}=4926 h^{-1}(\mu A)^{-1}$. Thus, $\gamma_{I} I_{*}=17241 h^{-1}$. A more accurate solution can be always obtained by using Newton iterative method for solving transcendental equations. This concludes the first step of testing.

At the second step, tests at two relative humidity levels $H_{1}$ and $H_{2}$, were conducted for the same temperature and voltage levels. This led to the relationship:

$$
\begin{aligned}
& \gamma_{H}=\frac{k T}{H_{1}-H_{2}}\left[\ln \left(-0.5800 \times 10^{-4} \frac{\ln P_{1}}{t_{1}}\right)-\right. \\
& \left.-\ln \left(-0.5800 \times 10^{-4} \frac{\ln P_{2}}{t_{2}}\right)\right]
\end{aligned}
$$

Let, e.g., after $t_{1}=40 \mathrm{~h}$ of testing at the relative humidity of $H_{1}=0.5$ at the given voltage (say, $\mathrm{V}=600 \mathrm{~V}$ ) and temperature (say, $T=60^{\circ} \mathrm{C}=333^{\circ} \mathrm{K}$ ), $5 \%$ of the tested modules failed, so that $P_{1}=0.95$, and after $t_{2}=55 \mathrm{~h}$ of testing at the same temperature and at the relative humidity of $\mathrm{H}_{2}=0.85,10 \%$ of the tested modules failed, so that $P_{2}=0.9$. Then the above equation, with the Boltzmann constant $k=8.61733 \times 10^{-5} \mathrm{eV} / \mathrm{K}$, yields: $\gamma_{H}=0.03292 \mathrm{eV}$.

At the third step, FOAT at two different voltage levels $V_{1}=600 \mathrm{~V}$ and $V_{2}=1000 \mathrm{~V}$ have been carried out for the same temperature-radiation bias, say, $T=85^{\circ} \mathrm{C}=358^{\circ} \mathrm{K}$ and $H=0.85$, and it has been determined that $10 \%$ of the tested devices failed after $t_{1}=40 h$ of testing $\left(P_{1}=0.9\right)$ and $20 \%$ of devices failed after $t_{2}=80 h$ of testing $\left(P_{2}=0.8\right)$. The voltage sensitivity factor can be found then as

$$
\begin{aligned}
& \gamma_{V}=\frac{0.02870}{400}\left[\ln \left(-0.5800 \times 10^{-4} \frac{\ln P_{2}}{t_{2}}\right)-\right. \\
& \left.-\ln \left(-0.5800 \times 10^{-4} \frac{\ln P_{1}}{t_{1}}\right)\right]=4.1107 \times 10^{-6} \mathrm{eV} / \mathrm{V}
\end{aligned}
$$

After the sensitivity factors of the leakage current, the humidity and the voltage are found, the stress free activation energy can be determined on the basis of the equation (14) for the given temperature and for any combination of loadings (stimuli). The third term in the equation (14) plays the dominant role, so that, in approximate evaluations, only this term could be considered.

Calculations indicate that the loading free activation energy in the above numerical example (even with the rather tentative, but still realistic, input data) is about $U_{0}=0.4770 \mathrm{eV}$. This result is consistent with the existing experimental data. Indeed, for semiconductor device failure mechanisms the activation energy ranges from 0.3 to $0.6 \mathrm{eV}$, for metallization defects and electromigration in $\mathrm{Al}$ it is about $0.5 \mathrm{eV}$, for charge loss it is on the order of $0.6 \mathrm{eV}$, for $\mathrm{Si}$ junction defects it is $0.8 \mathrm{eV}$.

\section{POSSIBLE NEXT GENERATION OF QT}

The next generation of EP QT could be viewed as a "quasi-FOAT," "mini-FOAT", a sort-of an "initial stage of FOAT" that more or less adequately replicates the initial non-destructive, yet full-scale, stage of FOAT. The duration and conditions of such a "mini-FOAT" QT could and should be established based on the observed and recorded results of the actual FOAT, and should be limited to the stage when no failures, or a predetermined and acceptable small number of failures in the actual full-scale FOAT, were observed. PHM technologies ("canaries") could and should be concurrently tested to make sure that the safe limit is established correctly and is not exceeded.

Such an approach to qualify EP devices into products will enable the industry to specify, and the manufacturers to assure, a predicted and adequate PoF for an EP product that passed the QT and is expected to be operated in the field under the given conditions for the given time. FOAT should be thoroughly designed, implemented, and analyzed, so that the QT is based on the trustworthy FOAT data. Since FOAT cannot do without simple, easy-to-use and physically meaningful PM, the role of such modeling, both computeraided and analytical (mathematical), in making the suggested new approach to QT practical and successful. It is imperative that the reliability physics that underlies the mechanisms and modes of failure is well understood. Such an understanding can be achieved only provided that flexible, powerful and effective PDfR efforts are implemented. 


\section{CONCLUSION}

The application of the PDfR concept and particularly the multi-parametric BAZ model enables one to improve dramatically the state of the art in the field of the EP products reliability prediction and assurance.

\section{REFERENCES}

[1] Suhir, E, "When Reliability is Imperative, Ability to Quantify It is a Must", IMAPS Advanced Microelectronics, July-August (2012)

[2] Suhir, E., Mahajan, R., "Are Current Qualification Practices Adequate?“, Circuit Assembly, April (2011)

[3] Suhir, E., 'Considering Electronic Product's Quality Specifications by Application(s)", Chip Scale Reviews, vol.16, No.4 (2012)

[4] Suhir, E, "Remaining Useful Lifetime (RUL): Probabilistic Predictive Model", International Journal of PHM, vol 2(2), (2011)

[5] Suhir, E, "Accelerated Life Testing (ALT) in Microelectronics and Photonics: Its Role, Attributes, Challenges, Pitfalls, and Interaction with Qualification Tests", Keynote address at the SPIE's 7-th Annual Int. Symp. on Nondestructive Eval. for Health Monitoring and Diagnostics, 17-21 March, San Diego, CA, (2002)

[6] Suhir, E, "Reliability and Accelerated Life Testing", Semiconductor International, February1, (2005)

[7] Suhir, E, Bechou, L., "Availability Index and Minimized Reliability Cost", Circuit Assemblies, February (2013)

[8] Suhir, E, "Thermal Stress Modeling in Microelectronics and Photonics Packaging, and the Application of the Probabilistic Approach: Review and Extension”, IMAPS International Journal of Microcircuits and Electronic Packaging, vol.23, No.2 (2000) (invited paper)

[9] Suhir, E, "Thermo-Mechanical Stress Modeling in Electronics and Photonics", Electronic Cooling, vol.7, No.4 (2001)

[10] Suhir, E "Thermal Stress Failures: Predictive Modeling Explains the Reliability Physics Behind Them", IMAPS Advanced Microelectronics, vol.38, No.4, July/August (2011)

[11] Suhir, E, "Predictive Modeling is a Powerful Means to Prevent Thermal Stress Failures in Electronics and Photonics", Chip Scale Reviews, vol.15, No.4, July-August (2011)

[12] Suhir, E, "Thermal Stress Failures in Electronics and Photonics: Physics, Modeling. Prevention", Journal of Thermal Stresses, June 3, (2013)

[13] Suhir, E, “Analytical Modeling in Structural Analysis for Electronic Packaging: Its Merits, Shortcomings and Interaction with Experimental and Numerical Techniques", ASME Journal of Electronic Packaging, vol. 111, No. 2, June (1989).

[14] Suhir, E, "Analytical Stress-Strain Modeling in Photonics Engineering: Its Role, Attributes and Interaction with the Finite-Element Method", Laser Focus World, May (2002).
[15] Suhir, E, "Analytical Thermal Stress Modeling in Electronic and Photonic Systems", ASME Applied Mechanics Reviews, invited paper, vol.62, No.4, (2009).

[16] Suhir, E, "Predicted Reliabilty of Aerospace Electronics: Application of Two Advanced Probabilistic Concepts", IEEE Aerospace Conference, Big Sky, Montana, March (2013)

[17] Suhir, E, "Probabilistic Design for Reliability", Chip Scale Reviews, vol.14, No.6 (2010)

[18] Suhir, E Mahajan, R., Lucero, A., Bechou, L., "Probabilistic Design for Reliability (PDfR) and a Novel Approach to Qualification Testing (QT)", 2012 IEEE/AIAA Aerospace Conf., Big Sky, Montana, (2012)

[19] E. Suhir, "Could Electronics Reliability Be Predicted, Quantified and Assured?" Microelectronics Reliability, No. 53, April 15, 2013

[20] Harry, M, Schroeder, R., "Six Sigma: The Breakthrough Management Strategy Revolutionizing the World's Top Corporations", Reed Business Information, Inc., (1999)

[21] Suhir, E., Bensoussan, A., Nicolics, J., Bechou, L., "Highly Accelerated Life Testing (HALT), Failure Oriented Accelerated Testing (FOAT), and Their Role in Making a Viable Device into a Reliable Product", 2014 IEEE Aerospace Conference, Big Sky, Montana, March 2014

[22] Suhir, E, "Failure-Oriented-Accelerated-Testing (FOAT) and Its Role in Making a Viable IC Package into a Reliable Product", Circuit Assembly, June (2013)

[23] E. Suhir,. and A. Bensoussan, "Quantified Reliability of Aerospace Optoelectronics," SAE Int. J. Aerosp. 7(1), 2014

[24] Suhir, E, "Analysis of a Pre-Stressed Bi-Material Accelerated Life Test (ALT) Specimen", Zeitschrift fur Angewandte Mathematik und Mechanik (ZAMM), vol.91, No.5, (2011)

[25] E. Suhir and J. Nicolics, "Analysis of a Bow-Free PreStressed Test Specimen", ASME JAM, vol.81, No.11, 2014 [26] Zhurkov, S.N., "Kinetic Concept of the Strength of Solids", Int. J. of Fracture Mechanics, vol.1, No.4, (1965)

[27] E. Suhir, A.Bensoussan, "Application of MultiParametric BAZ Model in Aerospace Optoelectronics", 2014 IEEE Aerospace Conference, Big Sky, Montana, March 2014

[28] Suhir, E, and Kang, S., "Boltzmann-ArrheniusZhurkov (BAZ) Model in Physics-of-Materials Problems", Modern Physics Letters B (MPLB), vol.27, April (2013)

[29] Suhir, E, Bechou L., and Bensoussan, A., "Technical Diagnostics of Electronics Products: Application of Bayes Formula and Boltzmann-Arrhenius-Zhurkov Model", Circuits Assembly, December 3 (2012)

[30] Suhir, E, "Applied Probability for Engineers and Scientists”, McGraw-Hill, New York, (1997)

[31] Suhir, E., "Statistics- and Reliability-Physics-Related Failure Processes", Modern Physics Letters B (MPLB), Vol. 28, No. 13, 2014 
[32] Suhir, E and Poborets, B., "Solder Glass Attachment in Cerdip/Cerquad Packages: Thermally Induced Stresses and Mechanical Reliability", Proc. of the 40th Elect. Comp. and Techn. Conf., Las Vegas, Nevada, May (1990); see also: ASME Journal of Electronic Packaging, vol. 112, No. 2, (1990).

\section{ACRONYMS}

$\mathrm{AT}=$ Accelerated testing;

$\mathrm{BAZ}=$ Boltzmann-Arrhenius-Zhurkov model;

BIT=Burn-in testing;

$\mathrm{CTE}=\mathrm{Coefficient}$ of thermal expansion (contraction);

$\mathrm{DfR}=$ Design for reliability;

$\mathrm{EP}=$ Electronics and photonics;

FOAT $=$ Failure oriented accelerated testing;
HALT=Highly accelerated life testing;

$\mathrm{MTTF}=$ Mean time to failure;

$\mathrm{MTTR}=$ Mean time to repair;

$\mathrm{OE}=$ Optoelectronics;

PDfR=Probabilistic DfR;

PDT $=$ Product development testing;

$\mathrm{PHM}=$ Prognostics and health monitoring;

$\mathrm{PM}=$ Predictive modeling;

$\mathrm{PoF}=$ Probability of failure;

$\mathrm{QT}=$ Qualification testing;

RUL=Remaining useful lifetime;

$\mathrm{SF}=$ Safety factor;

$\mathrm{SM}=$ Safety margin;

$\mathrm{TTF}=$ Time to failure;

Table 1. Accelerated test (AT) types

\begin{tabular}{|c|c|c|c|c|c|}
\hline ATtype & $\begin{array}{c}\text { Product development } \\
\text { testing (PDT) }\end{array}$ & $\begin{array}{l}\text { Highlyaccelerated } \\
\text { life testing (HALT) }\end{array}$ & $\begin{array}{l}\text { Qualification testing } \\
\text { (QT) }\end{array}$ & $\begin{array}{c}\text { Burn-in } \\
\text { testing (BT) }\end{array}$ & $\begin{array}{l}\text { Failure oriented } \\
\text { accelerated testing } \\
\text { (FOAT) }\end{array}$ \\
\hline Objective & $\begin{array}{l}\text { Technicalfeedback to } \\
\text { assure that the taken } \\
\text { design approach is } \\
\text { acceptable }\end{array}$ & $\begin{array}{l}\text { Ruggedize the } \\
\text { productand to } \\
\text { assess the } \\
\text { reliability limits }\end{array}$ & $\begin{array}{l}\text { Proof of reliability; } \\
\text { demonstration that } \\
\text { the product is } \\
\text { qualified to serve in } \\
\text { the given capacity }\end{array}$ & $\begin{array}{l}\text { Eliminate the } \\
\text { infant- } \\
\text { mortality part } \\
\text { of the bathtub } \\
\text { curve }\end{array}$ & $\begin{array}{l}\text { Understand the physics } \\
\text { of failure, confirm the } \\
\text { use of a particular } \\
\text { predictive model, } \\
\text { assess the probability } \\
\text { of failure }\end{array}$ \\
\hline End point & $\begin{array}{l}\text { Type, time, level, } \\
\text { andlor the number of } \\
\text { obsewed failures }\end{array}$ & $\begin{array}{c}\text { Predetermined } \\
\text { number or percent } \\
\text { of failures }\end{array}$ & $\begin{array}{l}\text { Predetermined time } \\
\text { andlor cycles, } \\
\text { andlor excessive } \\
\text { (unexpected) } \\
\text { number of failures }\end{array}$ & $\begin{array}{l}\text { Predetermine } \\
\text { d time andlor } \\
\text { loading level }\end{array}$ & $\begin{array}{l}\text { Predetermined number } \\
\text { or percent (typically } \\
\mathbf{5 0} \% \text { ) of failures }\end{array}$ \\
\hline $\begin{array}{l}\text { Follow-up } \\
\text { activity }\end{array}$ & $\begin{array}{l}\text { Failure anabysis, } \\
\text { design decision }\end{array}$ & Failure analysis & Passffail decision & $\begin{array}{l}\text { Shipping of } \\
\text { sound devices }\end{array}$ & $\begin{array}{c}\text { Failure and } \\
\text { probabilistic anabyses } \\
\text { of the test data }\end{array}$ \\
\hline Idealtest & Specific definitions & \multicolumn{3}{|c|}{ No failures in a long time } & $\begin{array}{c}\text { Numerous failures in a } \\
\text { shorttime }\end{array}$ \\
\hline
\end{tabular}

